\title{
SEQUENCE ANALYSIS OF tfdA ALPHA GENE SCREENED FROM INDIAN
}

\section{FRESH WATER BODIES}

\section{L. Jisnani and S. S. Bawage}

Sindhu Mahavidyalaya, Nagpur, (M.S.), India. Corresponding author Email : mljisnani@gmail.com

\section{Abstract:}

2, 4-dichlorophenoxy acetic (2,4-D) and their derivatives are commonly used herbicides and their presence in water bodies can be attributed to run of water. Several bacterial species of Sphingomonas, Ralstonia, Pseudomonas, Burkholderia, andBradyrhizobium are capable of degradation of 2,4 â€-D by Ît ketoglutarate dependent 2, 4-D dioxygenase coded by tfdA gene. In the present study we analyze the sequences of $\mathrm{tfdA}$ alpha genes that were screened from various fresh water bodies using metagenomic approach. Sequences were analyzed for the nucleotide contents, multiple sequence alignment, similarity and conservation followed by splits tree analysis. This study further extends our previous findings to give an insight into the sequence variations of tfdA alpha gene.

\section{Keywords:}

Metagenomics, tfdA alpha gene, Bradyrhizobium sp., sequence analysis, Riverwater

\section{Introduction:}

2,4-Dichlorophenoxyacetic acid or popularly known as 2, 4-D is a synthetic auxin used plant tissue culture media but extensively used as herbicide. It has now become acommon soil and water contaminant. Biodegradation of herbicide 2, 4-D is mediated by a dioxygenase coded bytfd A like gene (tfdA $\hat{\mathrm{I}} \pm$ ), to be precise, it encodes $\hat{I} \pm$ ketoglutarate dependent 2, 4-D dioxygenase thatconverts 2,4-D into 2,4-dichlorophenol and succinate. Many bacterial species of Sphingomonas, Ralstonia, Pseudomonas, Burkholderia, andBradyrhizobium harbor tfdA or tfdA like gene[1][2]. We have screened tfdA gene from river 
samples and discussed the presence of tfdA in fresh water bodies is suggestive of herbicide contamination. In this study, we extended our previous findings[3]by analyzing the screened tfdA gene sequence diversity.

\section{Material and Method:}

2.1. Sequence retrieval Gene sequences for tfdA sequences accession number HM177448, HM177449, HM177450, HM177451 and HM177452 were retrieved from NCBI nucleotide database. 2.2. Sequence statistics and multiple sequence alignment Sequences were analyzed for nucleotide counts andmultiple sequence alignment using CLC bio sequence viewer 6.0.2 and statistical output was generated using Minitab 14. 2.3. Entropy plot The conservation of nucleotide positions among the sequences as derived from multiple sequence alignment was further studied by generating an entropy plot using BioEdit. 2.4.

Splits tree analysis The neighbor-joining tree derived from the multiple sequence alignment was resolved with splits tree at 1000 bootstrap (http://www.splitstree.org/).

\section{Result and Discussion:}

The sequences were assumed to be of varied length even though amplified from same primers, the differences in the lengths was not statistically significant. Also to maintain uniformity, the nucleotides counts were expressed in percent. The sequences were GC rich as since it comprised of average $62.67 \%$ of total nucleotides and the $\mathrm{G}$ and $\mathrm{C}$ percentage were $32.60 \%$ and $30.52 \%$, respectively. Also the A and $\mathrm{T}$ content had no significant difference. The conserved count is evident in the standard deviation bar, corroborating with the purine and pyrimidine content of the eachsequence (Figure 1). The insignificant difference in the counts of nucleotide can be resolved in the multiple sequence alignment, which depicts variations, consensus sequence and percentage conservation along the sequence length (Figure 2). The additions and deletions in the sequence lead to gaps in the multiple sequence alignment, is represented by 
entropy plot (Figure 3). The overall variation between the sequences can be conceded by phylogenetic tree. The splits tree resolves the conventional phylogenetic tree to represent the diversity between the tfdA alpha gene sequences, which may be due different sampling place or bacteria (Figure 4).

\section{Discussion:}

Analysis of gene sequences gives the extent and magnitude of diversity as a measure to characterize genes. Statistical analysis gives preliminaryinformation about the purine/pyrimidine content summarizes the nature of DNA sequence. The tfdA alpha genes we

\section{Conclusion:}

To explore the new genes using culture independent approach especially, environmental samples requires balance of primer bias (specificity) and degeneracy.The identified conserved sequences of tfdA alpha gene can be incorporated for designing degenerate or specific probe/primer. This study shows a simple and rapid sequence analysis approach. It also refines and extends of our previous findings and concludes diversity among all the tfdA alpha genes.

\section{Acknowledgement :}

The work was carried out under DBT funded project on Godavari River Polutiion

\section{Reference:}

Itoh K, Kanda R, Sumita Y, Kim H, Kamagata Y, et al. (2002) tfdA-like genes in 2,4-dichlorophenoxyacetic acid-degrading bacteria belonging to the Bradyrhizobium-Agromonas-Nitrobacter-Afipia cluster in alpha-Proteobacteria. Appl Environ Microbiol 68: 3449-3454. 
Kamagata Y, Fulthorpe RR, Tamura K, Takami H, Forney LJ, et al. (1997) Pristine environments harbor a new group of oligotrophic 2,4dichlorophenoxyacetic acid-degrading bacteria. Appl Environ Microbiol 63: 2266-2272.

Thaware R R, Meenakshi R. Mohadikar, Nag B. B. S. P., Jambhulkar V. S. and Jisnani M. L. (2011) Screening of tfdA alpha gene in Indian fresh water bodies using metagenomic approach. Asiatic Journal of Biotechnology Resources 2.
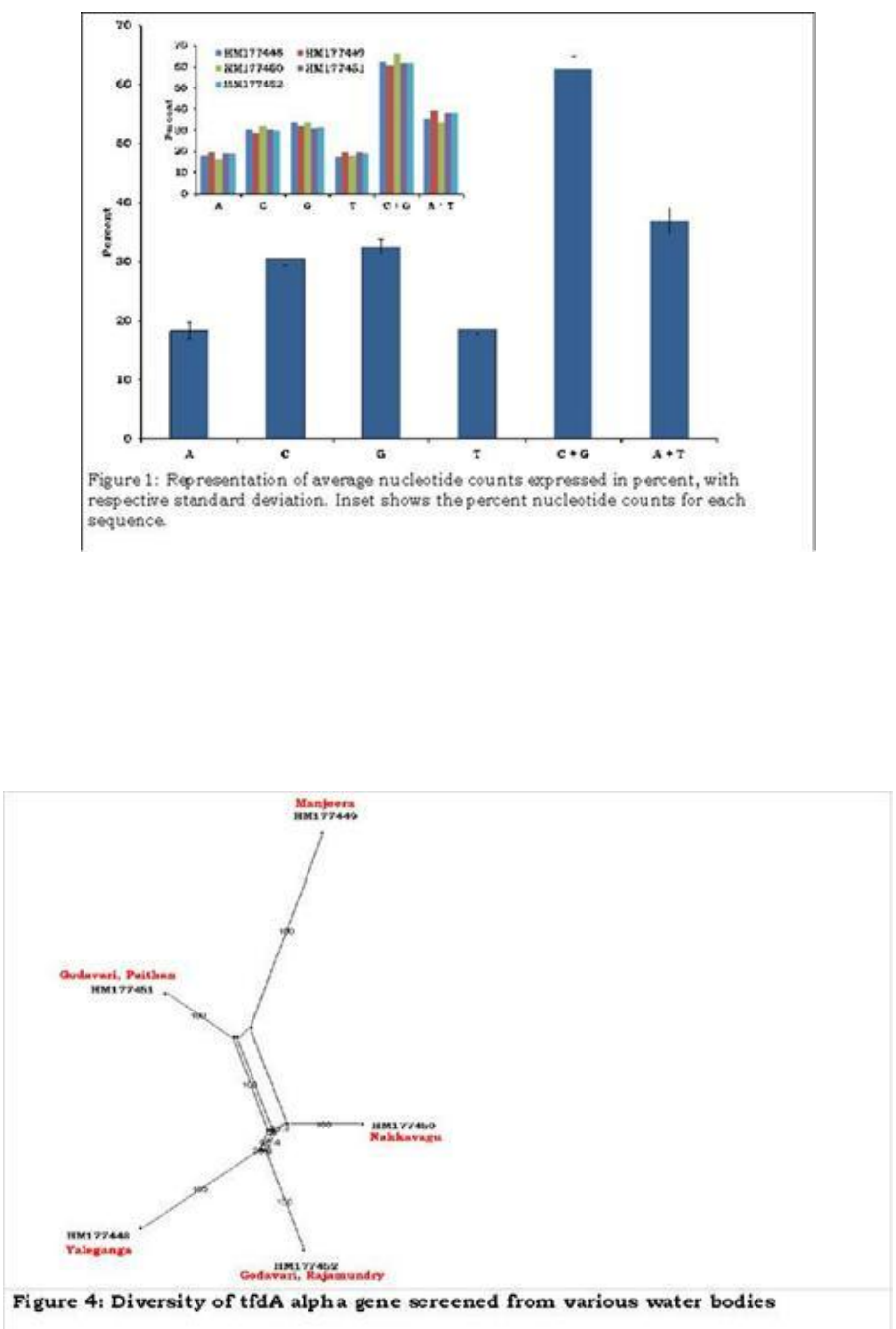


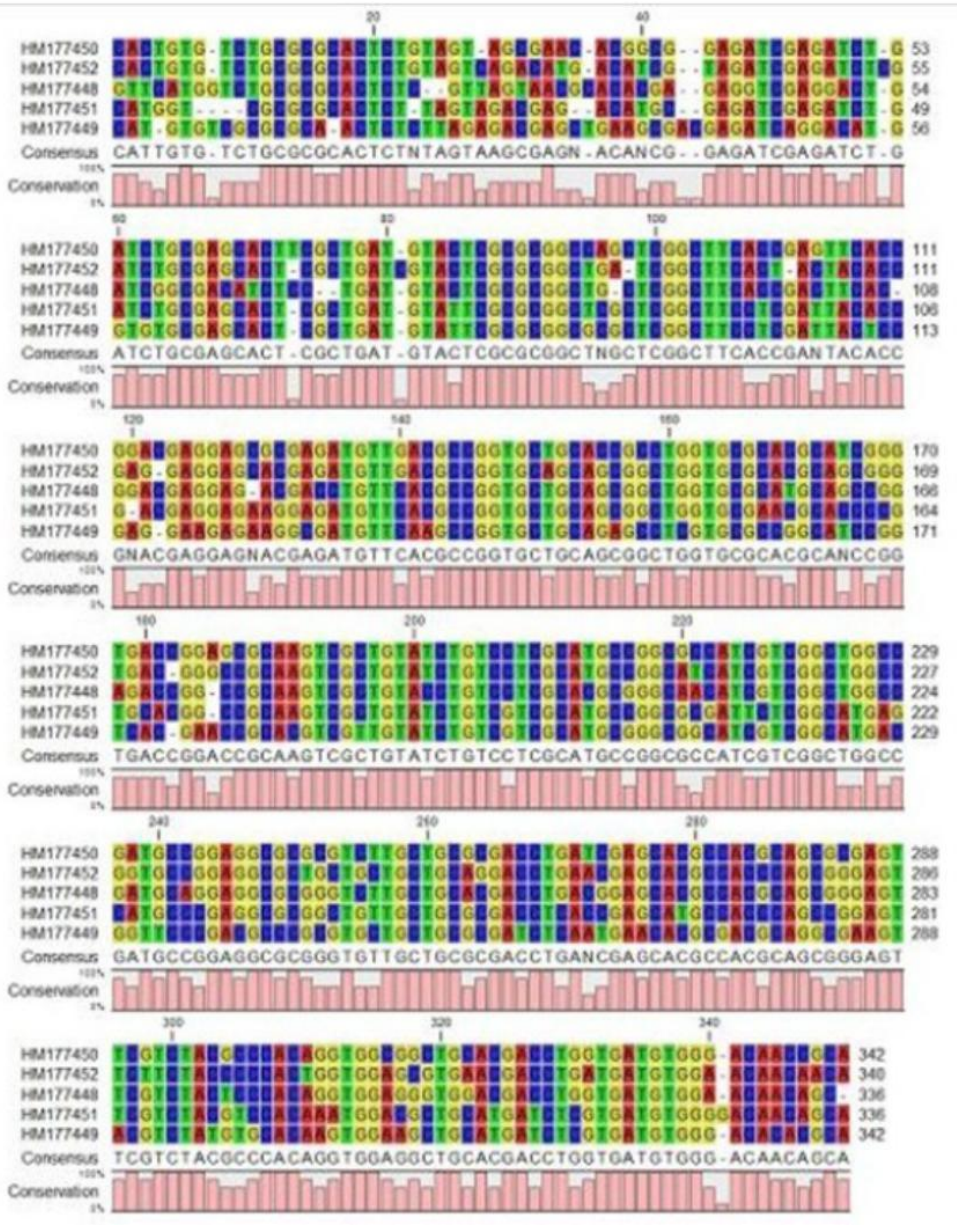

Figure 2: Multiple sequence alignment of tfdA alpha gene depicting variation, consensus sequence and percentage conservation..

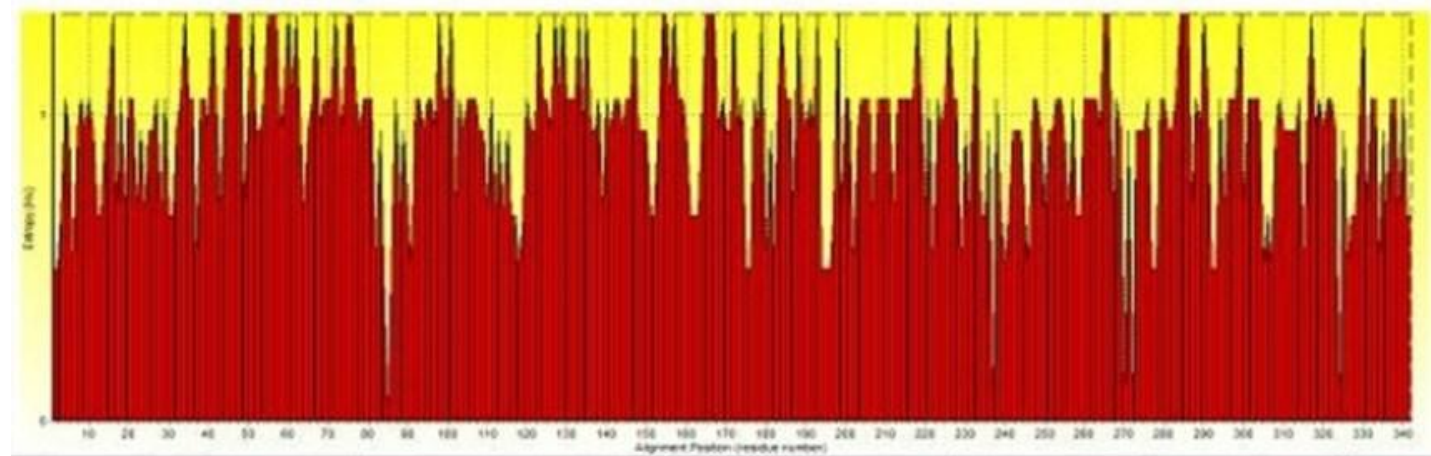

Figure 3 : Entropy plot representing the variation at respective nucleotide position 